\title{
How do paper and electronic records compare for completeness? A three centre study
}

\author{
Clara Hoi Ka Wu ${ }^{1}{ }^{1} \cdot$ Sheila M. H. Luk ${ }^{2} \cdot$ Richard L. Holder ${ }^{3}$ Zena Rodrigues ${ }^{4} \cdot$ Faisal Ahmed $^{4} \cdot$ lan Murdoch ${ }^{2}$
}

Received: 9 July 2017 / Revised: 15 February 2018 / Accepted: 16 February 2018 / Published online: 8 March 2018

(c) The Royal College of Ophthalmologists 2018

\begin{abstract}
Objectives Medical records are legal documentation of patients' care hence must be accurate and complete for both medical and legal purposes. Electronic patient record (EPR) systems aim to improve the accuracy of documentation, provide better organisation and access of data. This study compares the completeness of traditional note records and EPR in glaucoma patients.

Methods Using criteria from the April 2009 National Institute for Health and Care Excellence (NICE) guidelines completeness of data entry was compared between EPR and paper notes in three units. Moorfields Eye Hospital (City Road) uses the Openeyes EPR. Bedford Hospital (Moorfields Eye Centre) and Western Eye Hospital use the Medisoft EPR. The standard was set at $100 \%$ compliance for predetermined parameters.

Results One hundred seventy paper notes and 270 electronic records were analysed. With the exception of central corneal thickness $(p=0.31)$, all other key parameters were more consistently recorded in the paper records than in the EPR. Intraocular pressure $(p=0.004)$, anterior chamber configuration and depth assessments using gonioscopy $(p<0.001)$, fundus examination $(p=0.015)$, past medical history $(p<0.001)$, medication including glaucoma medication $(p<0.001)$ and drug allergies $(p<0.001)$.

Conclusions Our results show that paper records are significantly more complete than EPR. This is the case for two different EPRs and three separate sites. We propose additional training to aid data-collection; improving the design of EPRs by investigating factors such as layout and use of forced choice fields.
\end{abstract}

\section{Introduction}

Medical records are legal documentation of patients' care. As part of providing good clinical care, the General Medical Council (GMC) states that 'a clinician must keep clear, accurate and legible records, reporting the relevant clinical findings, the decisions made, the information given to patients, and any drugs prescribed or other investigations or treatment' [1]. The Francis Report highlighted that standard of record keeping was 'poor and inconsistent' [2]. This leads to

\footnotetext{
Clara Hoi Ka Wu

clarahoika.wu@gmail.com

1 Whittington Health NHS Trust, London, UK

2 Moorfields Eye Hospital NHS Foundation Trust, London, UK

3 Guy's and St Thomas' NHS Foundation Trust, London, UK

4 Imperial College Healthcare NHS Trust, London, UK
}

risk to patient safety and risk to Trust being found liable for mistakes that may be assumed to have occurred. It recommended that the standard of record-keeping should be audited regularly. Electronic patient record (EPR) systems aim to improve the accuracy of documentation, provide better organisation and access of data. Previous studies investigated the impact of electronic records on patient experience [3] and medication adherence [4]. To date, we have not found studies comparing the completeness of paper and electronic records in the diagnosis and management of glaucoma.

It has been estimated that 266,000 people are living with detected glaucoma in the United Kingdom [5]. Chronic open angle glaucoma (COAG) is a common form of glaucoma. It is usually asymptomatic until late stages of the disease where severe visual damage has occurred. Although COAG can occur with or without raised ocular pressure, ocular hypertension (OHT) remains one of the main risk factors for developing COAG [6].

National Institute for Health and Care Excellence (NICE) published guidelines on the diagnosis and 
Table 1 Essential assessments offered at diagnosis to those suspected of having COAG or those with OHT; and information recorded at the initial clinic visit

\begin{tabular}{|c|c|c|c|c|c|}
\hline & \multicolumn{2}{|l|}{ EPR } & \multirow{2}{*}{$\begin{array}{l}\text { EPR Total } \\
(n=270)(\%)\end{array}$} & \multirow{2}{*}{$\begin{array}{l}\text { Paper notes } \\
(n=170)(\%)\end{array}$} & \multirow[t]{2}{*}{ Chi-square $p$-value } \\
\hline & $\begin{array}{l}\text { Medisoft } \\
(n=170)\end{array}$ & $\begin{array}{l}\text { OpenEyes } \\
(n=100)\end{array}$ & & & \\
\hline Intraocular pressure & 168 & 90 & $258(95.6)$ & $170(100.0)$ & $0.004 *$ \\
\hline Central corneal thickness & 137 & 85 & $222(82.2)$ & $146(85.9)$ & 0.31 \\
\hline Gonioscopy & 106 & 64 & $170(63.0)$ & $151(88.8)$ & $<0.001$ \\
\hline Fundus examination & 151 & 69 & $220(81.5)$ & $153(90.0)$ & 0.015 \\
\hline Past medical history & 138 & 58 & $196(72.6)$ & $157(92.4)$ & $<0.001$ \\
\hline Current medications & 137 & 47 & $184(68.1)$ & $159(93.5)$ & $<0.001$ \\
\hline Glaucoma medication & 134 & 28 & $162(60.0)$ & $150(88.2)$ & $<0.001$ \\
\hline Drug allergies & 134 & 38 & $172(63.7)$ & 149 (87.6) & $<0.001$ \\
\hline
\end{tabular}

$P$-values obtained via chi-square tests and Fisher's exact test

*Fisher's exact test

management of COAG and OHT in April 2009. Around $9 \%$ of patients with OHT are at risk of developing COAG within 5 years. A diagnosis of COAG usually implies lifelong follow-up. Good record keeping (traditional notes or electronic) allows patients to be monitored against NICE standards in both hospital and community settings. This study aims to compare the completeness of traditional note records and EPR in glaucoma patients against NICE guidelines.

\section{Materials and methods}

NICE guidelines state that at diagnosis of patients who have or are suspected of having COAG or who have OHT should be offered the following essential tests:

- Intraocular pressure (IOP) measurement

- Central corneal thickness (CCT) measurement

- Peripheral anterior chamber configuration and depth assessment using gonioscopy

- Visual field measurement using standard automated perimetry

- Optic nerve and fundus assessment with dilatation using slit lamp biomicroscopy

The following should be recorded and available at each clinical episode:

- Past medical history including records of all previous tests and images relevant to COAG and OHT assessment

- Current systemic and topical medication

- Glaucoma medication

- Drug allergies and intolerances
Using criteria from the April 2009 NICE guidelines completeness of data entry was compared between EPR and paper notes in three units-Moorfield Eye Hospital (City Road), Western Eye Hospital and Bedford Hospital (Moorfields Eye Centre) during January 2010 to May 2015. Paper records were collected from Moorfield Eye Hospital and Western Eye Hospital; EPR records were collected from all three centres. Moorfields Eye Hospital (City Road) uses the Openeyes EPR. Western Eye Hospital and Bedford Hospital use the Medisoft EPR. Patients have either paper or electronic records filled in at first visit between the chosen timeframe, depending on clinician choice. Only electronic EPR was used for Bedford Moorfields. EPR was encouraged at City Road but it was clinicians' choice for which system to use. Either paper or electronic data was collected depending which system was chosen for that visit. Records from the initial clinical visit in patients with newly diagnosed glaucoma were analysed. The standard was set at $100 \%$ compliance with NICE guidelines for completeness. We chose to review the first 170 paper notes and the first 270 EPR in that timeframe who were newly diagnosed with OHT, COAG or suspected to have COAG. We collected the first 170 notes that were available. The list of EPR patients was generated by the electronic appointment system. Those patients who were not inappropriate e.g., not newly referred patients or with other diagnosis were then excluded.

All data was entered into a bespoke database and contingency tables were constructed and analysed with chisquare tests and Fisher's exact tests.

\section{Results}

One hundred seventy paper notes (Moorfields Eye Hospital, $n=100$; Western Eye Hospital, $n=70$ ) and 270 
electronic records (Moorfield Eye Hospital, $n=100$; Western Eye Hospital, $n=70$; Bedford Hospital, $n=100$ ) were analysed. All analyses were performed with the use of Microsoft Excel and Fisher exact tests.

With the exception of CCT, documentation of all other key parameters was significantly higher in paper records than in EPR-IOP (100 vs. 95.6\%, $p=0.004)$, anterior chamber configuration and depth assessments using gonioscopy ( 88.8 vs. $63.0 \%, p<0.001)$ and optic nerve and fundus assessment with dilatation using slit lamp biomicroscopy $(90.0$ vs. $81.5 \%, p=0.015)$ (Table 1$)$.

Information collected at the initial clinic visit was significantly more detailed in paper records compared to EPR-past medical history (92.4 vs. $72.6 \%, p<0.001)$, current systemic and topical medication (93.5 vs. $68.1 \%$, $p<0.001)$, glaucoma medication $(88.2$ vs. $60.0 \%$, $p<0.001)$ and drug allergies and intolerances $(87.6$ vs. $63.7 \%, p<0.001$ ) (Table 1).

Within electronic records, areas of poor documentation achieving less than $80 \%$ compliance were: anterior chamber configuration and depth assessments using gonioscopy (63\%), past medical history $(72.6 \%)$, current medication $(68.1 \%)$, glaucoma medication $(60.0 \%)$ and drug allergies and intolerances $(63.7 \%)$.

\section{Discussion}

Clinical governance involves continuously improving the quality of service and safeguarding high standards of care. All records should be reliable. Our study demonstrates that legible paper records are significantly more complete and reliable than EPR. This is the case for two different EPRs and three separate sites. A key measurement, visual field, was not currently electronically linked to the Openeyes EPR at Moorfields Eye Hospital, hence was excluded from the analysis. Findings from our study were similar to Linder et al. and Black et al. where there was no association between EPR use and good quality of care [7, 8]. Romano el al suggests that this is this case even with clinical decision support tools where reminders and alerts are adopted [9]. However, our results contrast sharply with those who investigated EPR use in diabetes care [10]. This may be because the study was carried out in context of a regional quality improvement collaborative and that clinicians who were using EPR were more willing to report their results.

Issues related to computer use are significant barriers to EPR [11]. A certain level of computer skill is required by clinicians in order to navigate through the electronic system. Many consider EPR challenging because of multiple screens and options. Therefore, insufficient technical knowledge can result in resistance. At Moorfields Eye Hospital and Western Eye Hospital, both paper and electronic records are used. Similar to Mikkelsen et al. [12], we found that when there is parallel use of two systems, this offers the preference to use one or the other. This deters from progressing to the sole use of EPR as it is natural to resist change. Another limitation would be that EPR is only recently introduced in glaucoma (2014) and our study is conducted during the switching over period to the new electronic system. In Bedford, however, the EPR is used uniquely and similar results were found for completion suggesting these factors are only part of the reason for poorer documentation.

The prompt access of patient information has huge advantages which are readily offered by electronic records. Big data allows storage, generation and synchronisation of large quantities of results [13]. This is now being used in Alzhelmer's disease and haematological cancers [14, 15]. Furthermore, compared to EPR, once clinical notes are lost, they cannot be recovered. As shown by Yoong et al., lost notes are associated with poor clinical outcome [16]. Our findings indicate that attention has to be paid to the completion and structure of records to ensure that they are fit for purpose. Additional points for consideration in the development of EPR include:

\section{Data entry correction}

Once saved, it can be difficult to revisit electronic records for correction. Since there is no cross-checking mechanism in place, it is difficult to ensure that data has been entered accurately. This has an impact on reviewing the data later on for clinical and research purposes. Incorrect records cannot be replaced despite being detected by data cleaning processes, whereas mistakes on written notes are easier to identify. In addition, errors in electronic prescribing were found to be unacceptably high by Theodossiades et al. [17]. Poor EPR data quality suggests that it is not yet mature enough to be a quality control system for health organisations on its own. Identifying reasons for poor compliance allows quality improvement.

\section{Reliability of records}

There are also concerns about the reliability of an electronic system [18]. Potential pitfalls include temporary loss of access to patient records if computer crashes or virus attack; or permanent loss of unsaved work in power failure. The recent NHS cyber-attack is an example where extensive disruption to hospital and general practice appointments has occurred [19]. 


\section{Medical ethics and law}

Confidentiality is one of the core values of ethical medical practice. 'Seeking a patient's consent to disclosure of information shows respect, and is part of good communication between doctors and patient' [20] Many are concerned about ethical issues associated with electronic records [21]. Data on the electronic system can be accessed by those who are not authorised to do so and inappropriate patient information disclosure can lead to legal consequences.

\section{Cost benefit analysis}

Information technology systems are often costly, but benefits are questionable. For example, the Department of Health initiatives of 'safer hospitals, safer wards; achieving an integrated digital care record' has cost the NHS $£ 500$ million since its publication in 2013 [22]. A recent study by Hakim et al. pointed out that the use of EPR may actually increase healthcare expenditure because clinicians with EPR access ordered more tests than those who did not have EPR access [23]. However, costs of ongoing training and maintenance of EPR compared to costs of storage and tracking written notes remain unclear and warrant further studies.

\section{Interference with doctor-patient relationship}

In Shachak's study, 92\% of clinicians felt that the use of EPR disrupted their communication with patients [24]. Morrison et al. suggests that EPR impedes the consultant's ability to conduct ward rounds [25]. Increase screen gaze time and inevitably less eye contact and conversation with patients negatively impacts on the doctor-patient relationship. In addition, the time gap between visualising the pathology and hunting for menus and buttons to record them can create opportunities for error. However, whether this really is a problem for doctors and patients require further research.

In order to fully adopt EPR in the future, we are discussing ways to improve the system. We are currently working on introduction of additional training on EPR for trainees. Furthermore, Riedmann et al. showed that decision support alerts reduce errors in prescribing [26]. These can easily be applied to electronic documentation. The layout of the electronic form can be similar to written proformas for ease of use. Finally, the use of forced choice fields will ensure key parameters are recorded. Further analysis is warrant to assess any improvement in the completion of EPR once electronic records are solely adapted.

In conclusion, our study shows paper records have better adherence in documentation about information required in the NICE guidelines. EPR has great potential for better data storage, processing and analysis. We propose additional training to aid data-collection; improving the design of EPRs by investigating factors such as layout and the use of forced choice fields.

\section{Summary}

\section{What was known before}

- Previous studies investigated the impact of electronic records on patient experience and medication adherence.

\section{What this study adds}

- To date, we have not found studies comparing the completeness of paper and electronic records in the diagnosis and management of glaucoma.

Acknowledgements The authors wish to acknowledge the assistance of Rui Feng, Sharmila Terkriwal and Zena Rodrigues in conducting research studies. We thank Pricilla Luk for her assistance in programming of patient data.

\section{Compliance with ethical standards}

Conflict of interest The authors declare that they have no conflict of interest.

\section{References}

1. General Medical Council. Record your work clearly, accurately and legibly. Good Med Pract. 2013; http://www.gmc-uk.org/guida nce/good_medical_practice/record_work.asp.

2. Francis R. Report of the Mid Staffordshire NHS Foundation Trust Public Inquiry Executive summary Report of the Mid Staffordshire NHS Foundation Trust Public Inquiry. 2013. p. 125.

3. Migdal CW, Namavar AA, Mosley VN, Afsar-manesh N. Impact of electronic health records on the patient experience in a hospital setting. J Hosp Med. 2014;9:627-33.

4. Delbanco T, Walker J, Bell SK, Darer JD, Elmore JG, Farag N, et al. Inviting patients to read their doctors' notes: A quasiexperimental study and a look ahead. Ann Intern Med. 2012;157:461-70.

5. Slade J. Eye health data summary. A review of published data in England February. 2014.

6. Kass MA, Heuer DK, Higginbotham EJ, Johnson CA, Keltner JL, Miller JP, et al. The Ocular Hypertension Treatment Study: a randomized trial determines that topical ocular hypotensive medication delays or prevents the onset of primary open-angle glaucoma. Arch Ophthalmol. 2002;120:701-13.

7. Linder J, Ma J, Bates DW, Middleton B, Stafford RS. ELectronic health record use and the quality of ambulatory care in the united states. Arch Intern Med. 2007;167:1400-5.

8. Black AD, Car J, Pagliari C, Anandan C, Cresswell K, Bokun T, et al. The impact of ehealth on the quality and safety of health care: a systematic overview. PLOS Medicine 2011; $8: 1-16$. 
9. Romano MJ, Stafford RS. Electronic health records and clinical decision support systems: impact on national ambulatory care quality. Arch Intern Med. 2011;171:897-903.

10. Cebul RD, Love TE, Jain AK, Hebert CJ. Electronic health records and quality of diabetes care. $N$ Engl $J$ Med. 2011;365:825-33.

11. Boonstra A, Broekhuis M. Barriers to the acceptance of electronic medical records by physicians from systematic review to taxonomy and interventions. BMC Health Serv Res. 2010;10:231.

12. Mikkelsen G, Aasly J, Engeset J, Naylor AR, Brook RH. Concordance of information in parallel electronic and paper based patient records. Int J Med Inform. 2001;63:123-31.

13. Auffray $\mathrm{C}$, Balling $\mathrm{R}$, Barroso $\mathrm{I}$, Bencze L, Benson $\mathrm{M}$, Bergeron J, et al. Making sense of big data in health research: Towards an EU action plan. Genome Med. 2016;8:71.

14. Geerts H, Dacks PA, Devanarayan V, Haas M, Khachaturian ZS, Gordon MF, et al. Big data to smart data in Alzheimer's disease: The brain health modeling initiative to foster actionable knowledge. Alzheimer's Dement. 2016;12:1014-21.

15. NICE. NICE plays key role in European project to help give better care to patients with blood cancers I News and features I News I NICE. https://www.nice.org.uk/news/article/nice-plays-key-rolein-european-project-to-help-give-better-care-to-patients-withblood-cancers Accessed date 13 July 2017.

16. Yoong A, Hudson C, Chard T. Medical audit: the problem of missing case-notes. Health Trends. 1993;25:114-6.

17. Theodossiades J, Shah S, Murdoch I. Anomalies in drug choice in glaucoma clinics. Eye. 2014;28:774-5.
18. Randeree E. Exploring physician adoption of EMRs: a multi-case analysis. J Med Syst. 2007;31:489-96.

19. BBC. NHS cyber-attack: GPs and hospitals hit by ransomware. BBC News. 2017; http://www.bbc.co.uk/news/health-39899646 Accessed date 10 July 2017.

20. General Medical Council, Medical School Council. Medical students: professional values. The duties of a doctor registered with the General Medical Council. 2009;1-66. http://www.gmc-uk.org/ Confidentiality__English_1015.pdf_48902982.pdf Accessed date 13 July 2017

21. Simon SR. Physicians and electronic health records. Arch Intern Med. 2007;167:507.

22. National Health Service England. High quality care for all, now and for future generations. Sign up to Saf. 2014; http://www.engla nd.nhs.uk/2014/06/24/sign-up-to-safety/ Accessed date 8 July 2017.

23. Hakim I, Hathi S, Nair A, Narula T, Bhattacharya J. Electronic health records and the frequency of diagnostic test orders. Am J Manag Care. 2017;23:e16-23.

24. Shachak A, Hadas-Dayagi M, Ziv A, Reis S. Primary care physicians' use of an electronic medical record system: a cognitive task analysis. J Gen Intern Med. 2017;24:341-8.

25. Morrison C, Jones M, Blackwell A, Vuylsteke A. Electronic patient record use during ward rounds: a qualitative study of interaction between medical staff. Crit Care. 2008;12:R148.

26. Riedmann D, Jung M, Hackl WO, Stühlinger W, van der Sijs H, Ammenwerth E. Development of a context model to prioritize drug safety alerts in CPOE systems. BMC Med Inform Decis Mak. 2011;11:35. 